Sebastiaan C. A. M. Bekkers

Walter H. Backes

Raymond J. Kim

Gabriel Snoep

Anton P. M. Gorgels

Valeria Lima Passos

Johannes Waltenberger

Harry J. G. M. Crijns

Simon Schalla

\section{Detection and characteristics of microvascular obstruction in reperfused acute myocardial infarction using an optimized protocol for contrast-enhanced cardiovascular magnetic resonance imaging}

Received: 12 March 2009

Revised: 8 May 2009.

Accepted: 22 May 2009

Published online: 9 July 2009

(C) The Author(s) 2009

This article is published with open access at Springerlink.com
S. C. A. M. Bekkers $(\bowtie)$.

A. P. M. Gorgels · V. L. Passos ·

J. Waltenberger - H. J. G. M. Crijns .

S. Schalla

Department of Cardiology,

Maastricht University Medical Center,

P. Debyelaan 25,

PO Box 5800, 6202 AZ

Maastricht, The Netherlands

e-mail: s.bekkers@mumc.nl

Tel.: +31-43-3875098

Fax: +31-43-3875104

W. H. Backes · G. Snoep

Department of Radiology,

Maastricht University Medical Center,

P. Debyelaan 25 ,

PO Box 5800, 6202 AZ

Maastricht, The Netherlands

\author{
R. J. Kim \\ Duke Cardiovascular Magnetic \\ Resonance Center, Duke University \\ Medical Center, \\ PO Box 3934 \\ Durham, NC 27710, USA
}

\begin{abstract}
Several cardiovascular magnetic resonance imaging (CMR) techniques are used to detect microvascular obstruction (MVO) after acute myocardial infarction (AMI). To determine the prevalence of MVO and gain more insight into the dynamic changes in appearance of MVO, we studied 84 consecutive patients with a reperfused AMI on average 5 and 104 days after admission, using an optimised single breath-hold 3D inversion recovery gradient echo pulse sequence (IR-GRE) protocol. Early MVO ( 2 min post-contrast) was detected in 53 patients $(63 \%)$ and late MVO (10 min post-contrast) in 45 patients $(54 \% ; p=0.008)$. The extent of MVO decreased from early to late imaging
\end{abstract}

$(4.3 \pm 3.2 \%$ vs. $1.8 \pm 1.8 \%, p<0.001)$ and showed a heterogeneous pattern. At baseline, patients without MVO (early and late) had a higher left ventricular ejection fraction (LVEF) than patients with persistent late MVO $(56 \pm 7 \%$ vs. $48 \pm 7 \%, p<0.001)$ and LVEF was intermediate in patients with early MVO but late MVO disappearance $(54 \pm 6 \%)$. During follow-up, LVEF improved in all three subgroups but remained intermediate in patients with late MVO disappearance. This optimised single breath-hold 3D IR-GRE technique for imaging MVO early and late after contrast administration is fast, accurate and allows detection of patients with intermediate remodelling at follow-up.

Keywords Acute myocardial infarction - Early and late microvascular obstruction - Contrastenhanced cardiovascular magnetic resonance imaging $\cdot 3 \mathrm{D}$ inversion recovery gradient echo pulse sequence $\cdot$ Ventricular remodelling

\section{Introduction}

Reperfusion therapy in patients with acute myocardial infarction (AMI) salvages myocardium and reduces mortality [1-3]. However, successful restoration of epicardial coronary artery patency after prolonged occlusion does not always lead to adequate reperfusion at the microvascular level. Previous studies have shown that the presence and absolute amount of microvascular obstruction (MVO) are associated with adverse left ventricular remodelling and prognosis [4-7]. Microvascular flow after reperfusion is spatially and temporally complex, with coexisting regions of hyperaemia, impaired vasodilatory flow reserve, low flow and no reflow. The magnitude and spatial extent of these perfusion patterns vary over time [8].

MVO can be detected with cardiovascular magnetic resonance imaging (CMR) using the technique of first pass perfusion imaging (FP) and traditional late contrastenhanced CMR (late CE-CMR), but no consensus exists on which technique is more accurate $[5,9-11]$. The 
importance of detecting MVO early after contrast injection is increasingly being recognised and has led to the development of additional CMR techniques [12, 13]. Whereas traditional late CE-CMR may underestimate the true extent of MVO as a result of diffusion of gadolinium into the area of MVO over time, FP allows only incomplete coverage of the left ventricle and has a lower signal-tonoise ratio and spatial resolution. When the technique of traditional late CE-CMR is performed early after contrast administration (early CE-CMR), the advantages of complete ventricular coverage, optimal signal-to-noise (SNR) and spatial resolution are combined and may overcome these shortcomings.

Optimal timing of imaging MVO and the dynamic changes of MVO over time in relation to clinical variables are not well known. In this study, a 3D inversion recovery pulse sequence was used early and late after contrast administration to determine the prevalence of MVO and gain more insight into the dynamic changes in appearance of MVO in relation to clinical variables. For early imaging the inversion time of the imaging sequence was first optimised in a pilot study.

\section{Materials and methods}

\section{Patients}

The study was approved by the institutional review board of our hospital, and patients were included after written informed consent was obtained. Between August 2006 and March 2008, 84 consecutive patients with a first ST segment elevation myocardial infarction (STEMI) referred to our institution for primary percutaneous coronary intervention (PCI) and presentation less than $12 \mathrm{~h}$ after symptom onset were prospectively studied. Definition of myocardial infarction (MI) was based on the recent consensus document including appropriate rise and fall in cardiac biomarkers [14]. Excluded were patients with claustrophobia, contraindications for CMR (cerebral clips, pacemakers, defibrillators, neurostimulators), severe congestive heart failure or cardiogenic shock (Killip class III and IV) at the time of CMR, atrial fibrillation, age below 18 years, severe renal failure (stage 4 or 5) and pregnancy.

Before emergency PCI all patients received aspirin (500 mg), heparin (5,000 U) and clopidogrel (600 mg). Use of thrombosuction and downstream administration of intravenous antiplatelet agents (abciximab), intracoronary nitroglycerine and adenosine were left to the discretion of the interventional cardiologist.

Cardiac biomarkers were sampled on admission, and at 6-, 12-, 18-, 24-, 36-, 48- and 72-h intervals. Area under the curve (AUC) biomarker levels were derived and expressed as $1,000 \mathrm{~h}$ units $\mathrm{l}^{-1}$.

\section{CMR protocol}

Patients underwent CMR imaging $5 \pm 2$ days (range 210 days) and $104 \pm 11$ days (range 88-122 days) after admission. Images were acquired during multiple breathholds on a 1.5-T MRI system (Philips Intera, Philips Medical Systems, Best, the Netherlands) with a cardiac software package and dedicated five-element phased array surface coil. ECG-gated cine images were obtained for functional analysis using a steady-state free precession sequence (slice thickness $6 \mathrm{~mm}$, slice gap $4 \mathrm{~mm}$, average repetition time (TR) and echo time (TE) $3.8 / 1.9 \mathrm{~ms}$, respectively, flip angle $50^{\circ}$, FOV $350 \mathrm{~mm}$, matrix $256 \times$ 256 , typically $22-25$ phases per cardiac cycle), in the following orientations: single slice two-chamber long axis, three-chamber long axis, four-chamber long axis, and a short axis stack covering the left ventricle (LV) from the base to apex (typically 10-14 slices).

Subsequently, a single breath-hold (15-20 s) 3D inversion recovery gradient echo sequence completely covering the LV was used for early and late CE-CMR (acquired slice thickness $12 \mathrm{~mm}$, reconstructed slice thickness $6 \mathrm{~mm}$, average TR/TE 3.9/2.4 ms, multishot (50 profiles/shot) segmented partial echo readout every heartbeat (TFE), flip angle $15^{\circ}$, FOV $400 \mathrm{~mm}$, matrix $256 \times 256$, acquired and reconstructed pixel size $1.56 \times 1.56 \mathrm{~mm}$, typically $16-18$ slices). The pulse sequence was run at 2 and $10 \mathrm{~min}$ after intravenous administration of $0.2 \mathrm{mmol} / \mathrm{kg}$ body weight gadolinium diethylenetriaminepentaacetic acid (Gd-DTPA, Magnevist ${ }^{\mathbb{R}}$, Schering, Germany; injection rate $3 \mathrm{ml} / \mathrm{s}$ ). For early CECMR, the inversion time was first optimised in a pilot study:

\section{Pilot study to find optimal TI for early CE-CMR}

We set out to find an optimal inversion time (TI) for early CE-CMR. For this, Look-Locker images acquired 2 min after intravenous administration of gadolinium $(0.2 \mathrm{mmol} / \mathrm{kg}$ body weight; injection rate $3 \mathrm{ml} / \mathrm{s})$ were quantitatively analysed in eight separate patients. This Look-Locker gradient echo (GRE) pulse sequence (slice thickness $10 \mathrm{~mm}$, average TR/TE $3.6 / 1.7 \mathrm{~ms}$, multishot (3 profiles/shot) segmented full echo readout every heartbeat, flip angle $8^{\circ}$, FOV $370 \mathrm{~mm}$, resolution $256 \times 256,39$ phases, phase interval $15 \mathrm{~ms}$ ) was applied at the short axis level with the most pronounced wall motion abnormalities. For analysis, epicardial and endocardial contours were manually drawn in each phase. Additional regions of interest (ROIs) were defined in: (1) a subendocardial hypoenhanced region (MVO), (2) a region adjacent to the hypoenhanced region (infarcted myocardium) and (3) noninfarcted (normal) myocardium (Fig. 1). All contours and ROIs were carefully adjusted for cardiac motion as a result of contraction and breathing. The signal intensities (SI) of MVO, infarcted and noninfarcted myocardium were 


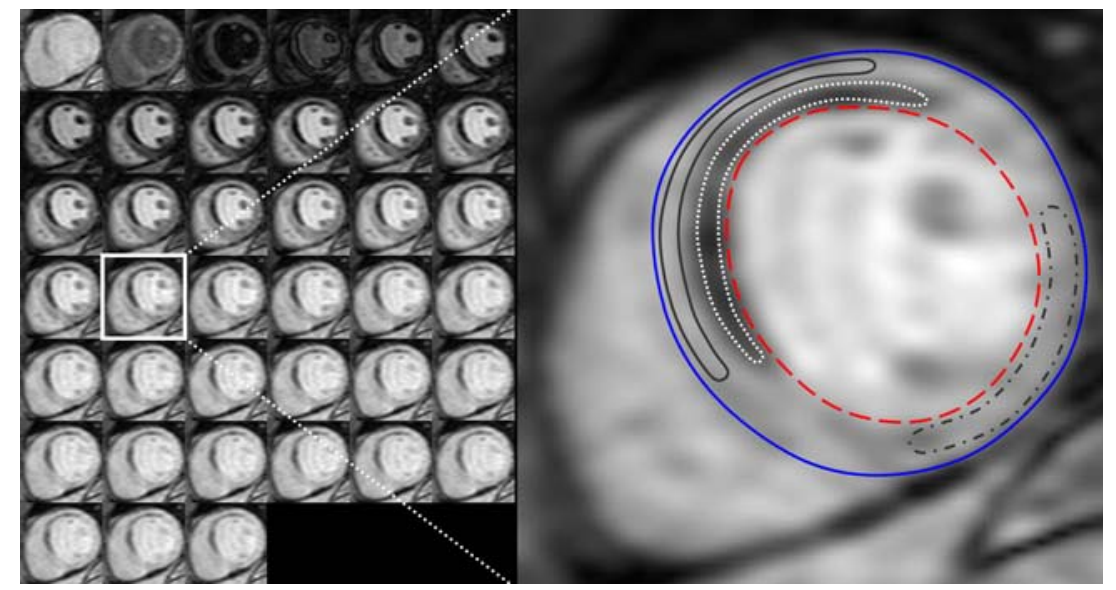

Fig. 1 Look-Locker images (left panel) in a patient with regions of interest (ROIs) drawn in only one phase (right panel, enlarged from left panel). Left panel 39 phases of a single slice Look-Locker sequence in one of the eight patients. Right panel phase 20 is enlarged to demonstrate the different ROIs that were drawn in all phases: subendocardial hypoenhanced region (MVO) (dotted line), region directly adjacent to the hypoenhanced region (infarcted myocardium) (solid black line), region of noninfarcted (normal) myocardium (dotted-dashed line) determined as a function of the Look-Locker TI (Fig. 2a). Measured inversion recovery SI curves were mathematically fitted to the inversion recovery magnetisation function, which provides estimates of the T1 relaxation time of these tissue regions [15]. T1 parameter estimations were calculated in the MATLAB (The MathWorks, Natick, MA) programming environment using a Levenberg-Marquardt nonlinear fitting algorithm. As the implemented Look-Locker pulse sequences provided magnitude images, the Rician noise distribution was taken into account [16]. Subsequently, the fitted SI curves of MVO and infarcted myocardium were subtracted to find the TI of maximum contrast, where contrast was defined as the difference in signal intensity (SI) between MVO and adjacent infarcted myocardium:

$$
\Delta \mathrm{SI}(\mathrm{TI})=\mathrm{SI}_{\text {infarct }}(\mathrm{TI})-\mathrm{SI}_{\mathrm{MVO}}(\mathrm{TI}) .
$$

The TI for which this contrast between MVO and adjacent infarcted myocardium was maximal would at first sight provide the optimal TI for early CE-CMR (Fig. 2a). However, a broad range of TI values (approximately 300 $700 \mathrm{~ms}$ ) were found with suboptimal overall signal characteristics of normal and infarcted myocardium. It was beneficial to use higher TI values, because higher signal levels will be acquired at nearly constant contrast, but with improved endocardial and epicardial border definition. This observation led us to define a merit function (MF), which was defined as the product of the signal difference between MVO and infarcted myocardium multiplied by the signal intensity of noninfarcted myocardium ( $\mathrm{SI}_{\text {normal }}$ ) (Fig. $2 b$ ):

$$
\mathrm{MF}(\mathrm{TI})=\left[\mathrm{SI}_{\text {infarct }}(\mathrm{TI})-\mathrm{SI}_{\mathrm{MVO}}(\mathrm{TI})\right] \cdot \mathrm{SI}_{\text {normal }}
$$

With this approach, both contrast and signal characteristics were simultaneously optimised. The merit function had a more narrow range of optimal TI values. The TI value of $530 \mathrm{~ms}(528 \pm 201 \mathrm{~ms})$, for which this merit function has its maximum, was used for subsequent early CE-CMR.

\section{Early CE-CMR}

With the inversion time (TI) set to $530 \mathrm{~ms}$, images showed increased signal intensity of the blood pool in the ventricular lumen, infarcted and noninfarcted myocardium. MVO was defined as a central hypoenhanced region within the area of the infarct-related artery (Fig. 3, left panel).

\section{Standard late CE-CMR}

Based on the results of a Look-Locker sequence run at 10 min after contrast injection (8 min after early CE-CMR), images were then acquired with an individually set inversion time (typical range $200-280 \mathrm{~ms}$ ) to optimally "null" noninfarcted myocardium. MVO was defined as a persistent central hypoenhanced area within the hyperenhanced area (Fig. 3, right panel).

\section{CMR image analysis}

CMR images were analysed off-line using commercially available software (CAAS MRV 3.0, Pie Medical Imaging, Maastricht, the Netherlands) blinded to clinical information. Endocardial and epicardial contours were manually traced in end-diastolic and end-systolic short axis slices to determine left ventricular end-diastolic volume (LVEDV) 


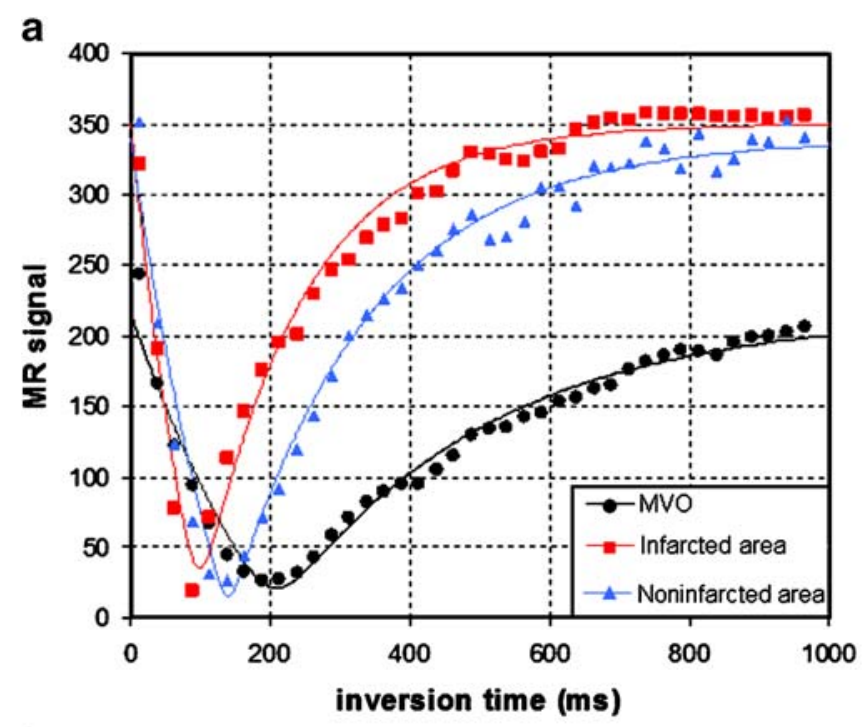

b

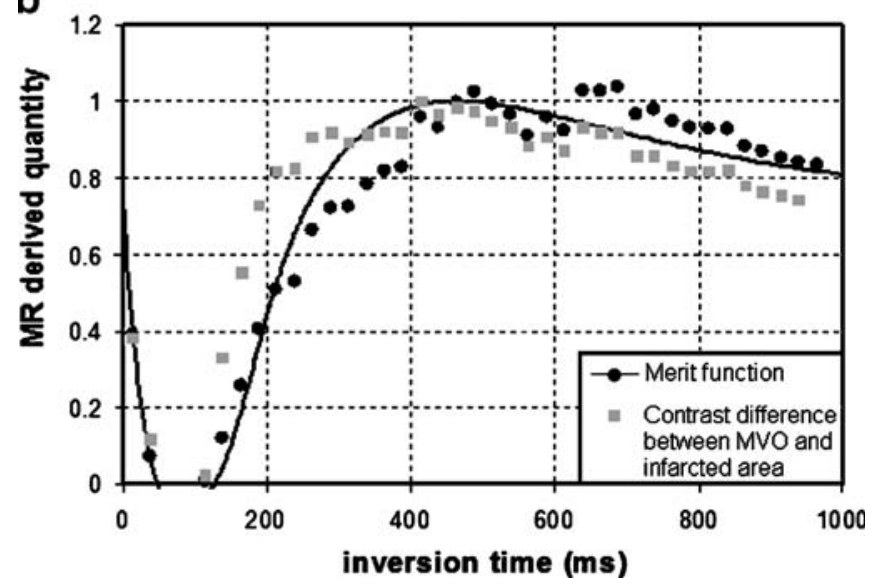

Fig. 2 Signal intensity curves obtained from the Look-Locker sequence (a) and merit function of contrast and SNR. The MRderived quantity was normalized to the maximum value (b). a Signal intensity (SI) curves in the region of microvascular obstruction (MVO), infarcted and noninfarcted myocardium obtained from Look-Locker inversion recovery images in a representative patient. Maximum contrast (i.e. SI difference) between MVO and infarcted myocardium would be obtained at an inversion time of approximately $340 \mathrm{~ms}$. b Combining optimised contrast and overall signal, the merit function provides a much less broader optimum at an inversion time of $465 \mathrm{~ms}$ for this case than for the contrast between MVO and infarcted area. Note the broad maximum in $\mathbf{b}$ and the relatively low signal at $340 \mathrm{~ms}$ (i.e. maximal SI difference in this case) in a

and left ventricular end-systolic volume (LVESV), left ventricular ejection fraction (LVEF), and left ventricular end-diastolic mass and were indexed to body surface area (BSA). Endocardial and epicardial contours were manually traced on both early and late CE-CMR images. The presence or absence of MVO was determined independently by two experienced observers. Infarct size was measured on the late CE-CMR images by manual planimetry of hyperenhanced areas (including central hypoenhancement if present) and expressed as a percentage of LV mass.

Statistical analysis

Continuous data are expressed as mean $\pm \mathrm{SD}$ or as median and range when non-normally distributed. Categorical data are expressed as frequencies with percentages. Differences between patients with and without early MVO were tested using an unpaired $t$ test. Spearman's rank correlation coefficient $(r)$ was used to determine correlations. The differences between proportions of paired categorical data were analysed using McNemar's test. All images were independently analysed by two observers blinded to the clinical data to determine the presence or absence of MVO, both for early and late. The interobserver agreement between readers was analysed by using kappa $(\kappa)$ statistics. The same statistics was used to check agreement between early and late MVO by one observer only. Unless otherwise stated, a two-tailed $p$ value less than 0.05 was considered statistically significant.

Depending on the MVO status, patients were classified into three groups: those without MVO on early and late CE-CMR (absence), those with MVO on both early and late CE-CMR (persistence), and those with MVO on early but not late CE-CMR (disappearance). Comparisons among these groups were conducted either with the parametric one-way ANOVA or the nonparametric Kruskal-Wallis test for continuous variables. Post hoc pairwise comparisons were Bonferroni corrected. For categorical variables Chi-squared or Fisher's exact tests were applied. Logistic univariable regression models were fitted to investigate which variables individually were significantly associated with presence of MVO (persistence or disappearance). Subsequently, only significant predictors (at a 0.1 significance level) were added to a multivariate model to estimate their independent/adjusted effects. Similarly, univariable logistic regression followed by multivariable analyses were conducted to detect independent predictors of MVO disappearance.

A paired sample $t$ test was used to investigate whether LVEDV index and LVEF significantly changed between baseline and follow-up measurements. Statistical analysis was performed with SPSS software (version 15.0 for Windows; SPSS Inc., Chicago, Illinois).

\section{Results}

The mean patient age was $60 \pm 11$ years and $61(73 \%)$ were men (Table 1). Most myocardial infarctions involved the right coronary artery $(55 \%)$, followed by the left anterior descending (32\%) and circumflex artery (13\%). More than half of the patients had single vessel disease (52\%). PCI with stenting was performed in 80 patients $(95 \%)$. In four 


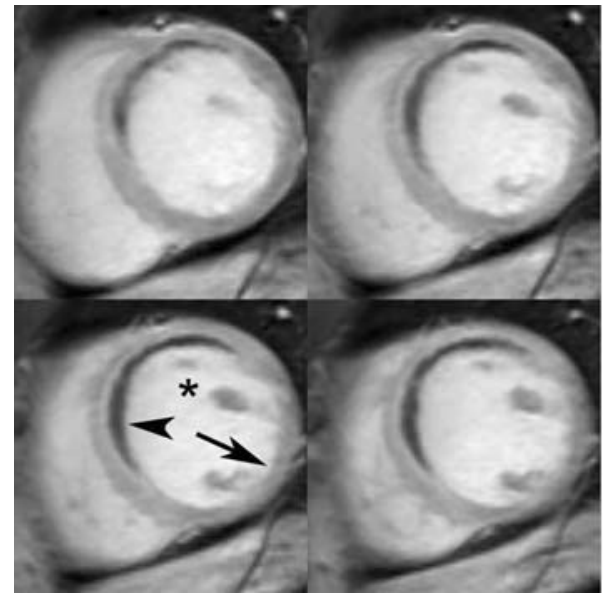

Fig. 3 Early (left panel) and late CE-CMR images (right panel) in one of the patients. Two sets of four contiguous short axis slices at midlevel of the LV in a patient 5 days after reperfused acute anteroseptal wall myocardial infarction. Left panel 2 min after contrast injection: the blood pool $(*)$ and myocardium show increased signal intensity (arrow), except for a central dark

patients no PCI was performed because of baseline thrombolysis in myocardial infarction (TIMI) 3 flow and a residual stenosis less than $30 \%$. Thrombosuction was performed in 26 patients (31\%), and intracoronary adenosine or nitroglycerine was given in 57 patients $(68 \%)$. Downstream abciximab was given in 43 patients (51\%). Final TIMI 3 flow was established in 75 patients (89\%) and TIMI 2 flow in the remaining 9 patients $(11 \%)$. The mean time from symptom onset to first balloon inflation or stent placement (total ischaemic time) was $219 \pm 85 \mathrm{~min}$. Mean CK area under the curve (AUC) was $54 \pm 37\left(1,000 \mathrm{~h}\right.$ units $\left.^{-1}\right)$. Mean infarct size was $16 \pm 11 \%$ of the LV mass.

\section{Early CE-CMR}

MVO on early CE-CMR was observed in 53 patients (63\%). The extent of MVO was $4.3 \pm 3.2 \%$ of the LV mass; expressed as absolute values this was $7.8 \pm 6.4 \mathrm{~g}$. Compared with patients with absence of MVO, those with MVO had a higher left ventricular end-systolic volume index (LVESVi) $\quad\left(45 \pm 13 \mathrm{ml} / \mathrm{m}^{2}\right.$ vs. $37 \pm 11 \mathrm{ml} / \mathrm{m}^{2}$, $p=0.008)$, lower ejection fraction $(49 \pm 7 \%$ vs. $56 \pm 7 \%$, $p<0.001)$, larger infarct size $(21 \pm 9 \%$ vs. $6 \pm 8 \%, p<0.001)$ and higher CK AUC $\left(68 \pm 32\right.$ vs. $30 \pm 31\left(1,000 \mathrm{~h}\right.$ units $\left.1^{-1}\right)$, $p<0.001)$. Left ventricular end-diastolic volume index (LVEDVi) and LV mass tended to be higher in patients with MVO but did not reach statistical significance $(86 \pm$ $17 \mathrm{ml} / \mathrm{m}^{2}$ vs. $81 \pm 14 \mathrm{ml} / \mathrm{m}^{2}, p=0.2$ and $64 \pm 12 \mathrm{~g} / \mathrm{m}^{2}$ vs. $59 \pm$ $10 \mathrm{~g} / \mathrm{m}^{2}, p=0.07$, respectively).

In the multivariable analysis with variables from Table 1 , only infarct size remained an independent predictor of the presence of MVO $(\exp \beta(\mathrm{OR}) 1.2,95 \% \mathrm{CI} 1.1-1.3$, $p<0.0001)$.

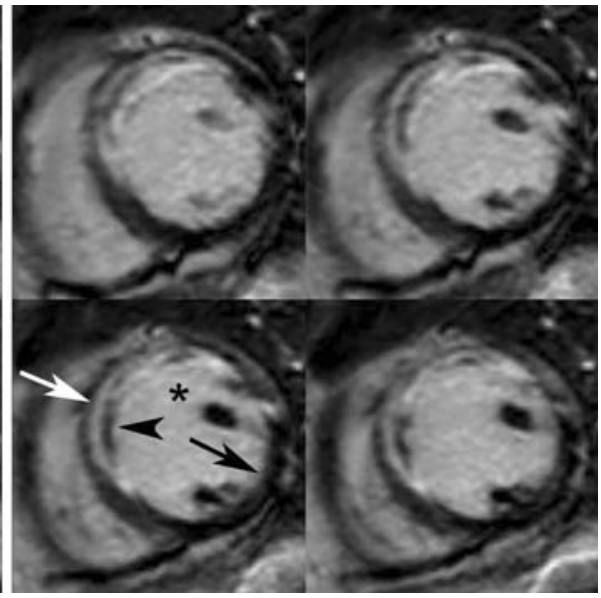

anteroseptal rim (arrowhead; early MVO). Right panel late hyperand hypoenhancement: $10 \mathrm{~min}$ after contrast injection, signal from noninfarcted myocardium is "nulled" (black arrow) and the infarcted myocardium is hyperenhanced (white arrow). A dark central rim is observed within the bright area (late MVO; arrowhead)

Table 1 Patient characteristics

\begin{tabular}{|c|c|}
\hline Characteristic & Value \\
\hline Age (years) & $60 \pm 11$ \\
\hline Male sex & $61(73)$ \\
\hline BMI & $27 \pm 4$ \\
\hline Smoking & $74(88)$ \\
\hline Hypercholesterolaemia & $30(36)$ \\
\hline Diabetes & $5(6)$ \\
\hline Previous angina pectoris & $38(45)$ \\
\hline \multicolumn{2}{|l|}{ Culprit } \\
\hline RCA & $46(55)$ \\
\hline LAD & $27(32)$ \\
\hline $\mathrm{Cx}$ & $11(13)$ \\
\hline \multicolumn{2}{|l|}{ Number of diseased vessels ${ }^{a}$} \\
\hline One & $44(52)$ \\
\hline Two & $19(23)$ \\
\hline Three & $21(25)$ \\
\hline Thrombosuction & $26(31)$ \\
\hline Time to balloon inflation (min) & $219 \pm 85$ \\
\hline Adenosine or nitroglycerine i.c. & $57(68)$ \\
\hline Abciximab downstream & $43(51)$ \\
\hline Final TIMI 3 flow & $75(89)$ \\
\hline CK (AUC, $1,000 \mathrm{~h}$ units $1^{-1}$ ) & $54 \pm 37$ \\
\hline Infarct size ( $\%$ of LV mass) & $16 \pm 11$ \\
\hline
\end{tabular}

Values are presented as number of patients with percentages in parentheses or mean \pm standard deviation

Abbreviations: i.c. intracoronary, $A U C$ area under the curve, $B M I$ body mass index, $R C A$ right coronary artery, $L A D$ left anterior descending artery, $C x$ circumflex artery

${ }^{a}$ Stenosis greater than $70 \%$ 
Table 2 Agreement between early and late MVO

\begin{tabular}{|c|c|c|c|}
\hline \multirow[t]{2}{*}{ Early MVO } & \multicolumn{3}{|c|}{ Late MVO } \\
\hline & Yes $(\%)$ & No $(\%)$ & Total (\%) \\
\hline Yes $(\%)$ & $45(54)$ & 8 & $53(63)$ \\
\hline No $(\%)$ & 0 & $31(37)$ & $31(37)$ \\
\hline Total $(\%)$ & $45(54)$ & $39(46)$ & 84 (100) \\
\hline
\end{tabular}

Values are presented as number of patients with percentages in parentheses

Good agreement was found between early and late MVO $(\kappa=0.8$, $p<0.001)$

\section{Late CE-CMR}

MVO on late CE-CMR (persistence of MVO) was observed in 45 patients (54\%) compared with $53(63 \%)$ patients with MVO on early CE-CMR $(p=0.008)$. Late MVO disappeared in eight patients $(9 \%)$ at the later time point. Late MVO was always found at the same location as early MVO (Fig. 3). The agreement between early and late MVO $(\kappa=0.8, p<0.001$, Table 2$)$ and the correlation between the extent of early and late MVO were good $(r=$ $0.8, p<0.0001$, Fig. 4). The interobserver agreement for the presence or absence of early and late MVO was excellent $(k$ value 0.9 for both early and late measurements, $p<0.001$ ).

\section{Early vs. late CE-CMR}

In all except one patient, the extent of MVO decreased significantly from early to late imaging $(4.3 \pm 3.2 \%$ vs. $1.8 \pm$ $1.8 \%, p<0.001$, Figs. 3 and 5). The median decrement in all patients was $66 \%$ (range $0-100 \%$ ); expressed as absolute amounts this resulted in a median decrement of $3.4 \mathrm{~g}$ (range $0-21 \mathrm{~g}$ ). The individual decrement was quite heterogeneous (Fig. 5).

Thus, early MVO was absent in 31 (37\%) patients, persisted in $45(54 \%)$ patients and disappeared in $8(9 \%)$ patients during late CE-CMR. Most baseline characteristics were not found to be related to absence, persistence and disappearance of MVO (Table 3). At baseline, infarct size and CK AUC were smallest and lowest in patients with absence of MVO, and largest for patients with persistence of late MVO $\left(30 \pm 31\right.$ vs. $73 \pm 32\left(1,000 \mathrm{~h}\right.$ units $\left.1^{-1}\right)$ and $6 \pm$ $8 \%$ vs. $23 \pm 9 \%, p<0.001$ for both). In addition, LVEF was highest in patients with absence of MVO, and lowest for patients with persistence of MVO (56 \pm 7 vs. $48 \pm 7 \%$, $p<0.001$ ).

The eight patients with disappearance of MVO had an infarct size and LVEF that was intermediate between those of patients with absence and persistence of MVO $(12 \pm 4 \%$ and $54 \pm 6 \%$, respectively, Table 3 ). These eight patients had a significantly smaller extent of early MVO than patients with persistent late MVO $(0.8 \pm 0.4 \%$ vs. $5 \pm 3.1 \%$, $p<0.001$, Fig. 5).
None of the clinical variables from Table 2 was found to be a significant predictor of MVO disappearance. From the CMR variables and after correction, only a smaller extent of early MVO remained independently associated with disappearance of late MVO $(\exp \beta(\mathrm{OR}) 0.034,95 \% \mathrm{CI}$ $0.001-0.908, p<0.04)$.

\section{Follow-up CMR}

LVEDV index decreased and LVEF increased in all three subgroups as compared with baseline (Table 3). LVEF remained highest in patients without MVO as compared with patients with persistence of late MVO $(59 \pm 6 \% \mathrm{vs}$. $51 \pm 7 \%, p<0.001)$. LVEF in patients with late MVO disappearance remained intermediate $(55 \pm 4 \%)$.

\section{Discussion}

The findings of this study in 84 patients with a reperfused first acute STEMI can be summarised as follows. First, the prevalence of MVO early after contrast injection at $2 \mathrm{~min}$ (early MVO) is higher than with traditional late CE-CMR at 10 min (late MVO) using an optimised single breath-hold $3 \mathrm{D}$ inversion recovery gradient echo technique. Second, the extent of MVO decreases significantly depending on the time of imaging after contrast administration and this decrease is quite heterogeneous (Figs. 3 and 5). Only the

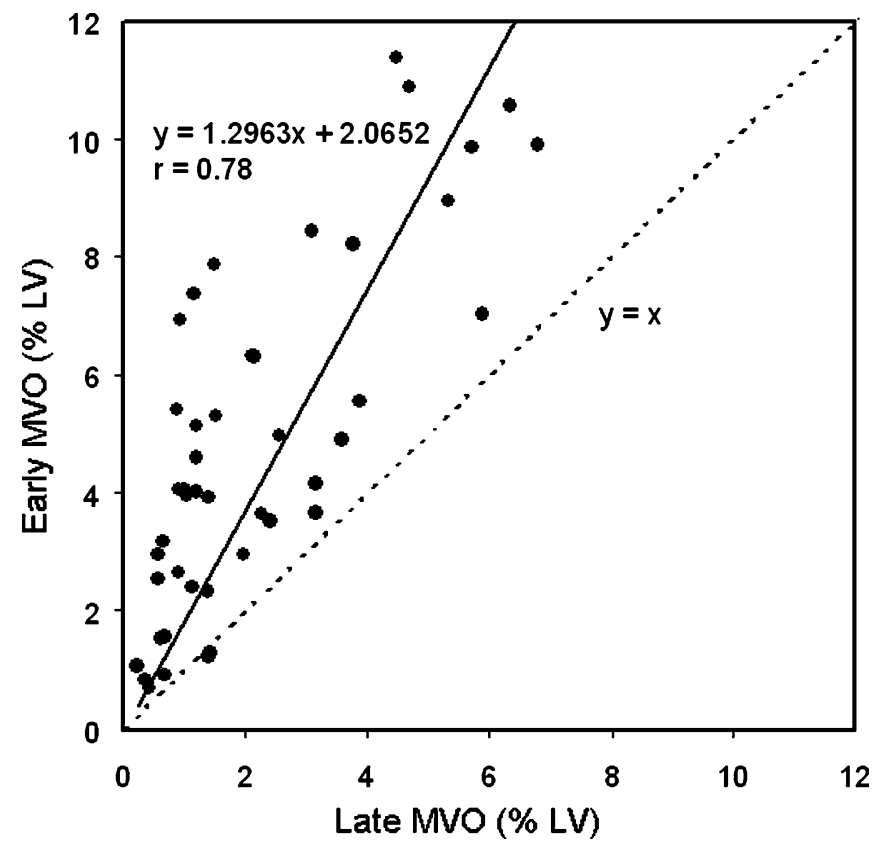

Fig. 4 Correlation between early and late MVO. A good correlation was found between the extent of early and persistent late MVO. The average extent of early MVO was larger than that of persistent late MVO $(r=0.78, p<0.001)$. The dotted line represents the line of identity and emphasises the inequality of the measures 


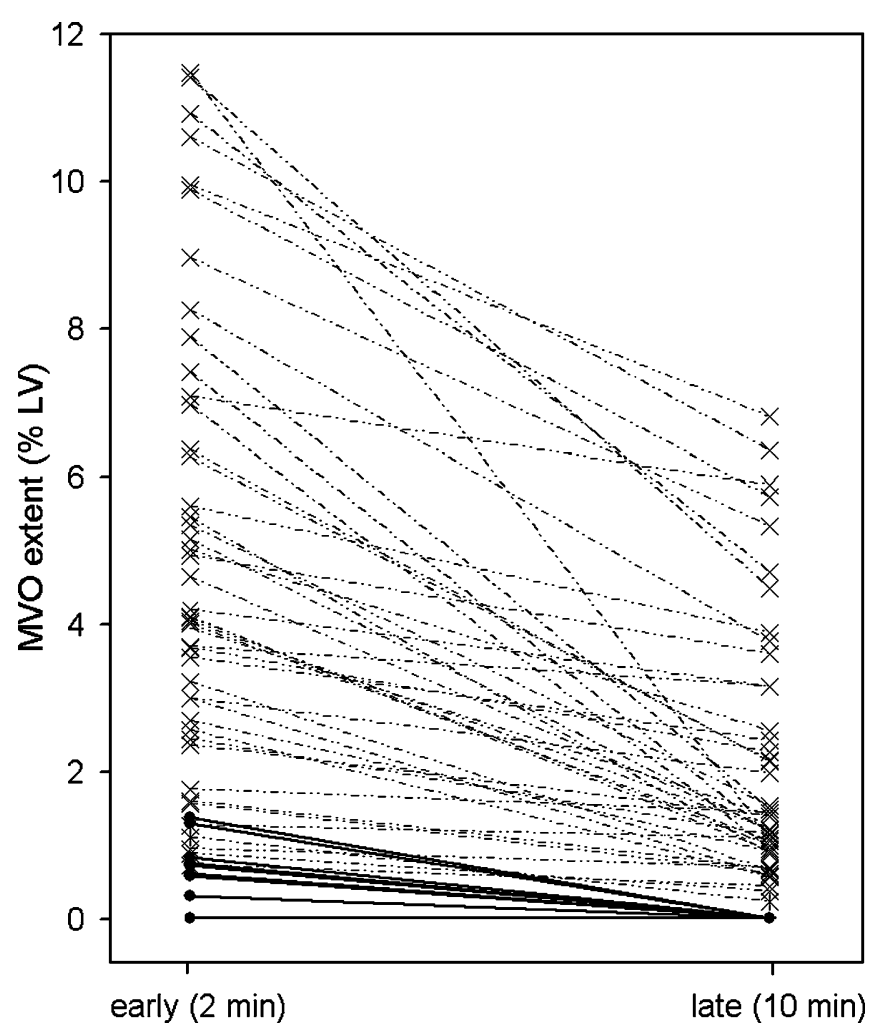

Fig. 5 Change in the extent of MVO. In all patients, the extent of early MVO decreased significantly but showed a heterogeneous pattern (MVO persistence $(\mathrm{x} \cdots \mathrm{x})$ ). In eight patients early MVO disappeared during late CE-CMR $(\bullet \bullet)$. These patients had a significantly smaller extent of early MVO than the patients with persistence of MVO $(0.8 \% \pm 0.4 \%$ vs. $5 \pm 3.1 \%, p<0.001)$

extent of early MVO is independently associated with persistence of late MVO. At baseline, patients with absence of MVO (early and late) have a significantly smaller infarct size and higher LVEF than patients with persistence of late MVO. In patients with early but late disappearance of MVO $(n=8)$, infarct size and LVEF are intermediate between those with absence and persistence of MVO and LVEF remains intermediate at follow-up.

The prevalence of MVO not only depends on the timing of imaging after infarction but also on the timing of imaging after contrast administration [9, 17]. In addition, it depends on the imaging technique used and as a consequence the reported prevalence of MVO has been inconsistent, ranging between 38 and $84 \%$ using FP and between 28 and $36 \%$ using traditional late enhancement imaging $[9,18]$. Although the numbers were not greatly different, we found that the prevalence of MVO using early CE-CMR at 2 min after contrast administration was significantly higher compared with late CE-CMR. Early MVO completely filled in during late CE-CMR in $10 \%$ of patients.

Despite the importance of detecting MVO in AMI patients, a 'gold standard' for the clinical detection of MVO has not been established yet. Several techniques measuring slightly different biological parameters are currently used (TIMI flow, blush grade, ST segment resolution, myocardial contrast echocardiography and scintigraphy). In addition, different CMR techniques are being used such as traditional late CE-CMR, contrast-enhanced cine MR imaging, T2-weighted imaging and FP, but the sensitivity is still undetermined [5, 9-11, 17, 19-22].

For the detection of early MVO, FP seems sensitive because of its histopathological correlation to MVO, as measured with thioflavin $\mathrm{S}$ staining and radioactive microsphere blood flow measurements in experimental studies [23]. FP, however, is hampered by incomplete left ventricular coverage, low SNR and low spatial resolution. Because of these shortcomings, other fast CMR techniques are increasingly being used for the detection of MVO early after contrast administration, including 3D T1-weighted inversion recovery gradient echo techniques [13, 24]. However, these studies did not specify the inversion times that were used for early imaging. Because we first optimised the inversion time for early imaging our approach allows imaging of the complete LV within a single breath-hold with good resolution, signal and contrast to noise.

As we used the same pulse sequence for early and late CE-CMR, except for different inversion times, this enabled us to accurately evaluate the dynamic changes in appearance of MVO over time. In all but one patient the extent of MVO significantly decreased from early to late imaging. We found a great heterogeneity in the shrinkage of MVO over time, indicating that the prevalence of MVO is only one part of the larger underlying pathophysiology of MVO (Figs. 3 and 5). For the first time we have described this heterogeneity in the shrinkage of MVO by using CMR. It likely represents the underlying heterogeneity in tissue perfusion, which was previously demonstrated in experimental studies. Negligible flow within the infarct core and progressively increasing flow towards the outer edges of the infarcted region are likely related to varying degrees of microvascular damage and obstruction $[23,25,26]$. Whether this relates to varying degrees of remodelling over time or carries adverse prognostic information is currently unknown and needs to be studied further.

Our protocol enabled us to distinguish an additional group of patients with early but disappearance of late MVO. Recently, Nijveldt et al. compared three CMR techniques (early, intermediate and late hypoenhancement imaging) but did not specifically describe this subgroup [13]. They found intermediate hypoenhancement in only two additional patients (3\%) compared with late hypoenhancement, whereas we observed this in eight additional patients $(10 \%)$. Although late hypoenhancement was the strongest predictor of $\mathrm{LV}$ remodelling over time in their study, we have shown that these patients have an LVEF that is intermediate at baseline and after follow-up. This may imply that these patients represent an intermediate risk group and should not be excluded from future therapies for MVO. 
Table 3 Patient characteristics in relation to MVO category (absence, disappearance and persistence)

\begin{tabular}{|c|c|c|c|c|}
\hline & \multicolumn{4}{|c|}{ Early MVO } \\
\hline & \multirow{2}{*}{$\begin{array}{l}\text { Absent } \\
(n=31)\end{array}$} & \multicolumn{2}{|l|}{ Present } & \multirow[b]{2}{*}{$p$ value } \\
\hline & & $\begin{array}{l}\text { Disappearance late MVO }(-) \\
(n=8)\end{array}$ & $\begin{array}{l}\text { Persistence late MVO }(+) \\
(n=45)\end{array}$ & \\
\hline Age (years) & $61 \pm 9$ & $61 \pm 10$ & $59 \pm 13$ & 0.8 \\
\hline Male sex & $22(71)$ & $5(63)$ & $34(76)$ & 0.7 \\
\hline BMI & $27 \pm 4$ & $26 \pm 2$ & $27 \pm 4$ & 0.7 \\
\hline Smoking & $26(84)$ & $7(88)$ & $41(91)$ & 0.6 \\
\hline Hypercholesterolaemia & $8(35)$ & $2(29)$ & $13(37)$ & 0.9 \\
\hline Diabetes & $3(10)$ & 0 & $2(4)$ & 0.4 \\
\hline Previous angina pectoris & $11(36)$ & $2(25)$ & $20(44)$ & 0.5 \\
\hline Anterior localisation of MI & $7(23)$ & $1(13)$ & $19(42)$ & 0.08 \\
\hline Three-vessel disease* & $8(26)$ & $1(13)$ & $12(27)$ & 0.7 \\
\hline Thrombosuction & $9(29)$ & $2(25)$ & $15(33)$ & 0.9 \\
\hline Time to balloon inflation (min) & $232 \pm 96$ & $196 \pm 80$ & $215 \pm 80$ & 0.6 \\
\hline Adenosine or nitroglycerine i.c. & $21(68)$ & $5(63)$ & $31(69)$ & 0.9 \\
\hline Abciximab downstream & $12(39)$ & $5(63)$ & $26(58)$ & 0.2 \\
\hline Final TIMI 3 flow & $27(90)$ & $5(71)$ & $41(91)$ & 0.3 \\
\hline Total CK (AUC, 1,000 h units $1^{-1}$ ) & $30 \pm 31^{\mathrm{a}}$ & $39 \pm 14^{\mathrm{b}}$ & $73 \pm 32^{\mathrm{a}, \mathrm{b}}$ & $<0.001^{\mathrm{a}} / 0.01^{\mathrm{b}}$ \\
\hline \multicolumn{5}{|l|}{ Baseline CMR } \\
\hline LVEDVi $\left(\mathrm{ml} / \mathrm{m}^{2}\right)$ & $81 \pm 14$ & $87 \pm 17$ & $86 \pm 17$ & 0.4 \\
\hline LVEF (\%) & $56 \pm 7^{\mathrm{c}}$ & $54 \pm 6$ & $48 \pm 7^{\mathrm{c}}$ & $<0.001^{\mathrm{c}}$ \\
\hline Infarct size (\% LV) & $6 \pm 8^{\mathrm{d}}$ & $12 \pm 4^{\mathrm{e}}$ & $23 \pm 9^{\mathrm{d}, \mathrm{e}}$ & $<0.001^{\mathrm{d}} / 0.003^{\mathrm{e}}$ \\
\hline Early MVO size ( $\%$ of LV) & $0^{\mathrm{f}}$ & $0.8 \pm 0.4$ & $5 \pm 3.1^{\mathrm{f}}$ & $<0.001^{\mathrm{f}}$ \\
\hline \multicolumn{5}{|l|}{ Follow-up CMR } \\
\hline LVEDVi (ml/m²) & $76 \pm 17$ & $85 \pm 16$ & $84 \pm 20$ & 0.2 \\
\hline LVEF (\%) & $59 \pm 6^{\mathrm{g}}$ & $55 \pm 4$ & $51 \pm 7^{g}$ & $<0.001^{\mathrm{g}}$ \\
\hline
\end{tabular}

Values are presented as number of patients with percentages in parentheses or mean \pm standard deviation

Abbreviations: BMI body mass index, i.c. intracoronary, $A U C$ area under the curve, $L V E D V i$ left ventricular end-diastolic volume index, $L V E S V i$ left ventricular end-systolic volume index, $L V S V i$ left ventricular stroke volume index, $L V E F$ left ventricular ejection fraction, $L V M i$ left ventricular mass index, $L E$ late enhancement, $M V O$ microvascular obstruction * Stenosis greater than $70 \%$

a,c,d,f,g For the difference between absence of MVO (early and late) and persistence of late MVO

${ }^{\mathrm{b}, \mathrm{e}}$ For the difference between disappearance and persistence of late MVO

There are some limitations to our study. The inversion times used for the 3D inversion recovery sequence of both early and late CE-CMR were determined with a LookLocker sequence. Although both sequences are gradient echo (GRE) readouts, the inherent difference in sequence parameters may cause differences in the amount of $\mathrm{T} 1$ weighting. It is therefore possible that the inversion times determined in our study are close but may be slightly out of kilter with the optimal inversion times for early and late CE-CMR. As the TI used in early CE-CMR was far above the "null" point for normal myocardium, small uncertainties in the optimal TI for early CE-CMR are not that critical as those for late CE-CMR. The optimal TI for late CECMR is traditionally set to "null" healthy myocardium 10 15 min after contrast administration in each patient. This is not possible for early CE-CMR, as the 2-min interval is too short for individual patients. Therefore, optimal TI for early CE-CMR was first determined in a pilot study.

The 2-min interval was chosen in line with histopathological validation of MVO in earlier studies [17, 23]. With even earlier imaging (i.e. $30 \mathrm{~s}$ ) sensitivity may increase but specificity may be decreased, because central hypoenhancement may appear in the setting of significant epicardial coronary disease even in the absence of myocardial infarction. Moreover, in our study we were interested in the dynamic change in appearance of MVO over time. Earlier imaging might have possibly shown a greater level of dynamic change. However, because most patients showed substantial shrinkage of MVO, the exact timing of early imaging is not expected to be that critical. 
In conclusion, using an optimised single breath-hold 3D inversion recovery gradient echo pulse sequence, the prevalence of MVO early after the injection of contrast medium is higher than with traditional late CE-CMR. Our results show that the appearance of MVO is dynamic and heterogeneous, and imaging MVO early and late after contrast administration may give more information about the severity and pathophysiology of MVO. In addition, this approach allows the discrimination of MVO subgroups that have different infarct sizes and remodelling parameters.

Open Access This article is distributed under the terms of the Creative Commons Attribution Noncommercial License which permits any noncommercial use, distribution, and reproduction in any medium, provided the original author(s) and source are credited.

\section{References}

1. The GUSTO investigators (1993) An international randomized trial comparing four thrombolytic strategies for acute myocardial infarction. N Engl J Med 329:673-682

2. Serruys PW, Simoons ML, Suryapranata H et al (1986) Preservation of global and regional left ventricular function after early thrombolysis in acute myocardial infarction. J Am Coll Cardiol 7:729-742

3. Sheehan FH, Doerr R, Schmidt WG et al (1988) Early recovery of left ventricular function after thrombolytic therapy for acute myocardial infarction: an important determinant of survival. J Am Coll Cardiol 12:289-300

4. Gerber BL, Rochitte CE, Melin JA et al (2000) Microvascular obstruction and left ventricular remodeling early after acute myocardial infarction. Circulation 101:2734-2741

5. Hombach V, Grebe O, Merkle N et al (2005) Sequelae of acute myocardial infarction regarding cardiac structure and function and their prognostic significance as assessed by magnetic resonance imaging. Eur Heart J 26:549-557

6. Ito H, Tomooka T, Sakai N et al (1992) Lack of myocardial perfusion immediately after successful thrombolysis. A predictor of poor recovery of left ventricular function in anterior myocardial infarction. Circulation 85:1699-1705

7. Wu KC, Zerhouni EA, Judd RM et al (1998) Prognostic significance of microvascular obstruction by magnetic resonance imaging in patients with acute myocardial infarction. Circulation 97:765-772

8. Villanueva FS (2002) Myocardial contrast echocardiography in acute myocardial infarction. Am J Cardiol 90:38J-47J

9. Lund GK, Stork A, Saeed M et al (2004) Acute myocardial infarction: evaluation with first-pass enhancement and delayed enhancement MR imaging compared with ${ }^{201}$ Tl SPECT imaging. Radiology 232:49-57
10. Taylor AJ, Al-Saadi N, Abdel-Aty H, Schulz-Menger J, Messroghli DR, Friedrich MG (2004) Detection of acutely impaired microvascular reperfusion after infarct angioplasty with magnetic resonance imaging. Circulation 109:2080-2085

11. Tarantini G, Cacciavillani L, Corbetti F et al (2005) Duration of ischemia is a major determinant of transmurality and severe microvascular obstruction after primary angioplasty: a study performed with contrast-enhanced magnetic resonance. J Am Coll Cardiol 46:1229-1235

12. Comte A, Kastler B, Laborie L, Hadjidekov G, Meneveau N, Boulahdour H (2008) Using a contrastenhanced imaging sequence at 3-minute delay in 3-T magnetic resonance imaging for acute infarct evaluation. Invest Radiol 43:669-675

13. Nijveldt R, Hofman MB, Hirsch A et al (2009) Assessment of microvascular obstruction and prediction of short-term remodeling after acute myocardial infarction: cardiac MR imaging study. Radiology 250:363-370

14. Thygesen K, Alpert JS, White HD et al (2007) Universal definition of myocardial infarction. Circulation 116:2634 2653

15. Haacke EMBR, Thompson MR (1999) Magnetic resonance imaging, physical principles and sequence design. Wiley, New York, pp 133-134

16. Karlsen OT, Verhagen R, Bovee WM (1999) Parameter estimation from Rician-distributed data sets using a maximum likelihood estimator: application to T1 and perfusion measurements. Magn Reson Med 41:614-623

17. Rochitte CE, Lima JA, Bluemke DA et al (1998) Magnitude and time course of microvascular obstruction and tissue injury after acute myocardial infarction. Circulation 98:1006-1014

18. Yan AT, Gibson CM, Larose E et al (2006) Characterization of microvascular dysfunction after acute myocardial infarction by cardiovascular magnetic resonance first-pass perfusion and late gadolinium enhancement imaging. J Cardiovasc Magn Reson $8: 831-837$
19. Gerber BL, Garot J, Bluemke DA, Wu KC, Lima JA (2002) Accuracy of contrast-enhanced magnetic resonance imaging in predicting improvement of regional myocardial function in patients after acute myocardial infarction. Circulation 106:1083-1089

20. Kramer CM, Rogers WJ Jr., Mankad S, Theobald TM, Pakstis DL, Hu YL (2000) Contractile reserve and contrast uptake pattern by magnetic resonance imaging and functional recovery after reperfused myocardial infarction. J Am Coll Cardiol 36:1835-1840

21. Raff GL, O'Neill WW, Gentry RE et al (2006) Microvascular obstruction and myocardial function after acute myocardial infarction: assessment by using contrast-enhanced cine MR imaging. Radiology 240:529-536

22. Stork A, Lund GK, Muellerleile K et al (2006) Characterization of the periinfarction zone using T2-weighted MRI and delayed-enhancement MRI in patients with acute myocardial infarction. Eur Radiol 16:2350-2357

23. Judd RM, Lugo-Olivieri CH, Arai $\mathrm{M}$ et al (1995) Physiological basis of myocardial contrast enhancement in fast magnetic resonance images of 2-dayold reperfused canine infarcts. Circulation 92:1902-1910

24. Bogaert J, Kalantzi M, Rademakers FE, Dymarkowski S, Janssens S (2007) Determinants and impact of microvascular obstruction in successfully reperfused ST-segment elevation myocardial infarction. Assessment by magnetic resonance imaging. Eur Radiol $17: 2572-2580$

25. Ambrosio G, Weisman HF, Mannisi JA, Becker LC (1989) Progressive impairment of regional myocardial perfusion after initial restoration of postischemic blood flow. Circulation 80:1846-1861

26. Kloner RA, Ganote CE, Jennings RB (1974) The "no-reflow" phenomenon after temporary coronary occlusion in the dog. J Clin Invest 54:1496-1508 